\title{
A fractal approach to the temporal distribution of microseismicity at the low eastern flank of Mt. Etna during 1989-1994
}

\author{
V. Latora ${ }^{\text {a,c }}$, A. Rapisarda ${ }^{\text {a,b, * }}, \mathrm{S}$. Vinciguerra ${ }^{\mathrm{d}, \mathrm{e}}$ \\ a Dipartimento di Fisica Universitá di Catania, Catania, Italy \\ ${ }^{\mathrm{b}}$ Istituto Nazionale di Fisica Nucleare, Sezione di Catania, Catania, Italy \\ ${ }^{\mathrm{c}}$ Laboratorio Nazionale del Sud, I.N.F.N. Catania, Italy \\ ${ }^{\mathrm{d}}$ Istituto di Geologia e Geofisica, Universitá di Catania, Catania, Italy \\ ${ }^{\mathrm{e}}$ Benfield Greig Hazard Research Centre, Department of Geological Sciences, University College London, London, UK
}

Received 2 January 1998; revised 6 April 1998; accepted 2 September 1998

\begin{abstract}
Seismicity affecting the low eastern flank of Mt. Etna shows clustering in time. We analysed a catalog of microearthquakes recorded by the four stations of the Osservatorio Sismologico di Protezione Civile di Acireale during the period 1989-1994. In general a strong deviation from periodic and random distribution was found. The temporal clustering is scale invariant and a fractal dimension can be derived to characterize it. Fractal dimensions seem to be correlated to the beginning of two major eruptions. (C) 1998 Elsevier Science B.V. All rights reserved.
\end{abstract}

Keywords: Fractal clustering; Microseismicity; Etna volcano

\section{Introduction}

During the last decade, several papers have appeared in the literature concerning time clustering of earthquakes in terms of fractal analysis. Smalley et al. (1987) demonstrated that this approach allows one to discriminate between random, periodic and clustered scale-invariant time distributions. Fractal analysis gives also quantitative information that can help in studying the underlying dynamical mechanisms of seismic processes. In this respect, it is of

\footnotetext{
* Corresponding author. Tel.: + 39-95-7195214; Fax: + 39-95383023; E-mail: rapisarda@ct.infn.it
}

great interest to apply this method to the seismic activity of the complex Etnean area.

In particular, in this paper, we considered the seismic activity at the low eastern flank of Mt. Etna volcano during 1989-1994. This area plays a key role in the geodynamic framework of the volcano. The temporal distribution of the seismic activity is controlled by different processes, such us tectonic forces, the magma uprising (two eruptions occurred during the period considered) and the gravitational spreading of the eastern flank of the volcano. The fractal analysis was performed in order to characterize quantitatively the seismic activity and to better understand how the latter is controlled by the different processes. 


\section{Fractal clustering}

In this Section the method for characterizing fractal clustering is briefly reviewed and a simple example is illustrated. The concept of fractal dimension was introduced by Mandelbrot (1967) to describe quantitatively objects that are not regular nor smooth, but present some roughness. Since then, this concept has found many applications in various fields of science.

In the following we introduce the so called 'boxcounting' definition for the fractal dimension. The box-counting dimension $D$ of a fractal object is defined as (Mandelbrot, 1967)

$N(l) \sim 1 / l^{D}$ for $l \rightarrow 0$,

$N(l)$ being the number of boxes of side length $l$ needed to cover the object. The total length of the object at the scale 1 is given by

$L(l)=l N(l)$.

Hence, substituting Eq. (1) in Eq. (2), one obtains

$L(l) \sim l^{1-D}$.

Therefore, by plotting $L(l)$ as a function of $l$ in a $\log -\log$ scale, it is possible to extract the fractal dimension $D$ from the slope of the resulting straight line. In general $D$ is equal to the usual topological dimension for regular objects, i.e., $D=0$ for a point, $D=1$ for a line and $D=2$ for a surface. For fractal objects, however, $D$ is greater than the topological dimension and it is a non-integer number.

As an example one can consider the case of coastline. For a rugged coastline like that of Great Britain one finds $D=1.25$ (Mandelbrot, 1967). Usually the relations (1) and (3) are satisfied only in a limited range of $l$-values due to the finite size of real objects.

In general, one can extend the concept of fractal dimension to probability distributions. In fact, suppose we have $M$ events distributed according to a given fractal pattern along a segment of total length $L_{\mathrm{tot}}$. In order to obtain information on this pattern, we study the distribution at different length scales. That is, we divide $L_{\text {tot }}$ into several small intervals of length $l$ and we count the number of intervals containing at least one event. We can define the occupancy probability $P$ as the ratio of filled intervals
$N(l)$ divided by the total number of intervals whose length is $l$, i.e., $N_{\text {tot }}(l)=L_{\text {tot }} / l$. Therefore we have

$P(l)=\frac{N(l)}{N_{\mathrm{tot}}(l)}=\frac{l N(l)}{L_{\mathrm{tot}}}$.

Note that $l N(l)$ is simply the total length of the filled intervals defined in Eq. (2). Hence substituting Eq. (3) into Eq. (4), one gets the relation between the occupancy probability and the fractal dimension of the distribution

$P(l)=\frac{L(l)}{L_{\mathrm{tot}}} \sim l^{1-D}$.

Thus

$D=1-\frac{\ln P(l)}{\ln l}$ for $l \rightarrow 0$.

If we plot $P(l)$ vs. $l$ in a $\log -\log$ scale for a fractal distribution we get a straight line from which the value of $D$ can be extracted.

A fractal distribution shows a typical clustering which is very different from that of a periodic or a random sequence. In general a distribution can be completely clustered if all events are concentrated in a very small time interval. In this case the fractal dimension coincides with the topological one and $D=0$. The opposite limiting case is for a periodic distribution, where the events are equally spaced in time. In this case there is no clustering at all. The fractal dimension coincides again with the topological one and $D=1$. For a random time sequence the distribution is centered around an average time interval. One gets $D=0$ for very small sampling time intervals and $D=1$ for sampling time intervals larger than the average one. In this case the eventual clustering observed at small time scales is not scale invariant. A temporal distribution which exhibits fractal clustering has a self-similar pattern characterized by $0<\mathrm{D}<1$ over a wide time scale.

A common problem in extracting the fractal dimension is the finite size of the data sets which one investigates. In fact the quantity $P(l)$ defined in Eq. (4) is a real occupancy probability only if a large set of data is considered. Thus, it is very important to have a precise quantitative indication of the error one obtains when the data set is limited. 


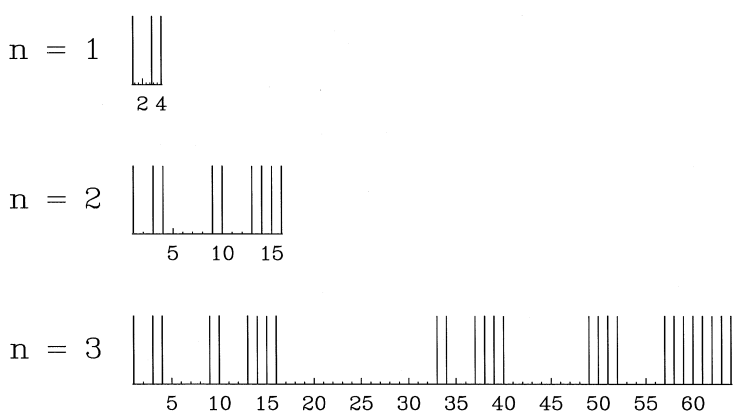

Fig. 1. Schematic picture of the first three steps for the Growing Asymmetric Cantor (GAC) set. See text for further details.

In order to illustrate this approach and mainly its limitations due to finite size effects, we study the fractal dimension of the Growing Asymmetric Cantor (GAC) set. The latter is a well known fractal object (Tél et al., 1989) having an entire spectrum of fractal dimensions. More properly it is a "multifractal' object, which complete spectrum of generalized dimensions can be determined analytically (Tél et al., 1989). It is therefore well suited for our purpose. In Fig. 1 we show the first three iterative steps to construct the GAC set. In the first step, we have 3 events distributed over an interval whose length is 4 , no events at the second position. In the second step,the distribution is the sum of the configuration for $n=1$ (which is the seed) plus a twice enlarged version of the seed, which starts at a position equal to twice the length of the seed configuration. At the nth step the fractal has a length equal to $4^{n}$ and contains $3^{n}$ events. The analytical value of the zeroth order fractal dimension is $D=0.6942 \ldots$ (Tél et al., 1989). We generated the GAC set up to the 4th and 5th order obtaining two sample distributions of total lengths 256 and 1024 with $M=81$ and $M=243$ events respectively. In Fig. 2 we plot $\ln (P(l))$ as a function of $\ln (l)$ for the 4 th (a) and the 5 th (b) order of the GAC set. The curves are indicated with the letter $\mathrm{F}$ for fractal. Large fluctuations and a saturation for very small and very large values of $\ln (l)$ are evident. Both effects are due to the limited number of events considered and in fact diminish by increasing the order of the iteration. However, if one makes a linear fit in a region that excludes the lower and the upper saturation ranges, one obtains a fractal dimension, according to Eq. (6), which is very close to the analytical one within the errors of the fit. Of course the longer the sequence the smaller the error. More precisely, we get

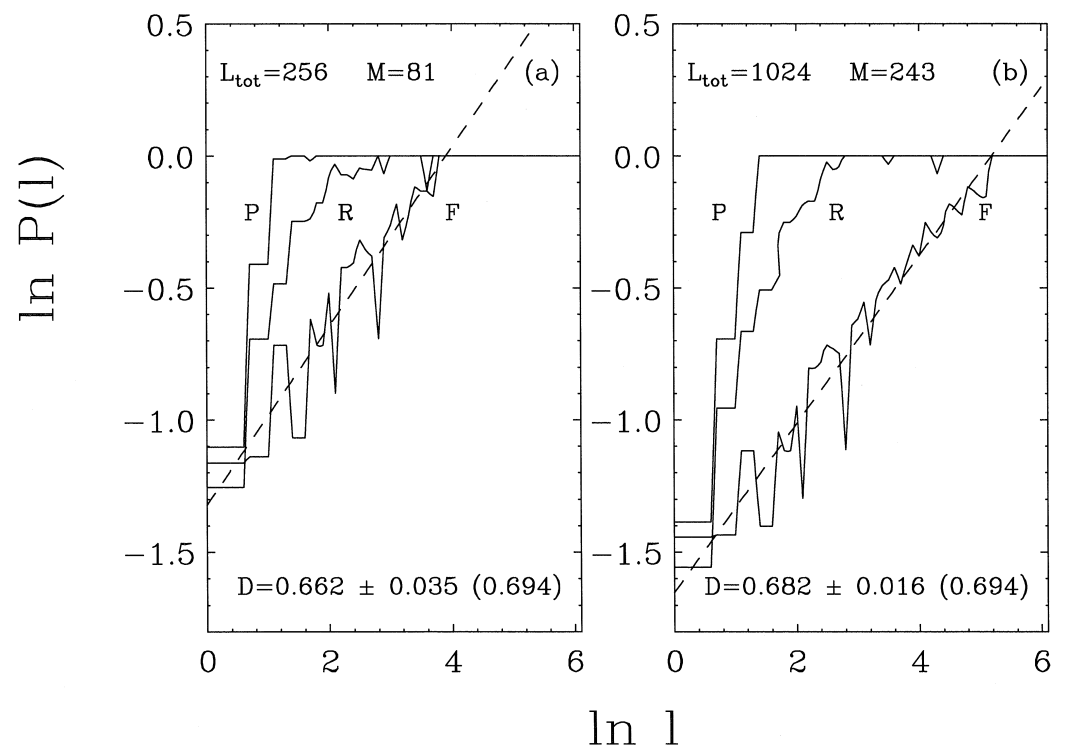

Fig. 2. We plot $\ln (P(l))$ as a function of $\ln (l)$ for the 4th (a) and the 5th (b) order of the GAC set. The curves which refer to the fractal set are indicated with the letter F. The dashed lines are linear fits which allow to extract the fractal dimension $D=0.662 \pm 0.035$ for the 4 th order iteration and $D=0.682 \pm 0.016$ for the 5 th order iteration respectively. In parenthesis we report the exact value. The curves corresponding to periodic and uniform random distributions are indicated with $\mathrm{P}$ and $\mathrm{R}$ respectively. 
$D=0.662 \pm 0.035$ for the 4 th order iteration and $D=0.682 \pm 0.016$ for the 5 th order iteration respectively. The fits are shown as dashed lines in Fig. 2. In the same figure two more curves are reported. They correspond to a periodic and a uniform random distribution of the $M$ events over the total length of the interval. They are indicated respectively with $\mathrm{P}$ for periodic and $\mathrm{R}$ for random. In both cases, i.e., $M=81$ and $M=243$, the periodic and the random distribution are easily distinguishable from the scale invariant fractal one.

In the following we apply the above discussed fractal approach to a temporal distribution of $M$ earthquakes occurring in a time interval whose total length is $T$. We studied $\ln (P(\tau))$ as a function of $\tau$, $\tau$ being a small time interval. The total length of the time intervals and the total number of events are very similar to those used for the 4 th and 5 th order GAC set. Therefore the limited size of the distribution should not prevent a reasonable determination of $D$, if a fractal clustering exists. The approach should also be able to discriminate between a periodic and a random sequence of events.

This kind of analysis was applied for the first time by Smalley et al. (1987) to sequences of earthquakes as a function of time. They demonstrated that the New Hebrides seismicity between mid-1978 and mid-1984 shows clear signs of scale-invariant fractal clustering in time. The latter characterizes these temporal distributions in a quantitative way and allows to discriminate between periodic and random artificial sequences of records.

Evidence of fractal clustering in earthquakes temporal distribution have been recently reported also by Kagan and Jackson (1991). These authors proposed another quantitative measure of the degree of clustering based on the study of the distribution of the intercurrence time $\Delta$ between two events. They defined the coefficient of variation $C$ as

$$
C=\frac{\sigma}{\langle\Delta\rangle}
$$

$\langle\Delta\rangle$ being the average intercurrence time between two events and $\sigma$ being the standard deviation of this interval distribution.

In general, for a random distribution the probability of having a time interval $\Delta$ between two events
Table 1

Values of $M, D,\langle\Delta\rangle, \sigma$ and $C$ for the GAC set of different length with and without a random additive noise

\begin{tabular}{lcrrrr}
\hline$\delta_{\text {noise }}$ & $M$ & $D$ & $\langle\Delta\rangle$ & $\sigma$ & $C$ \\
\hline GAC set & $L_{\text {tot }}=256$ & & \\
0 & 81 & $0.662 \pm 0.35$ & 3.2 & 8.3 & 2.6 \\
\multicolumn{7}{l}{ GAC set } & $L_{\text {tot }}=1024$ & & & \\
0 & 243 & $0.682 \pm 0.16$ & 4.2 & 19.6 & 4.6 \\
$10 \%$ & 266 & $0.6 \pm 0.05$ & 3.8 & 9.7 & 2.5 \\
$20 \%$ & 291 & $0.58 \pm 0.05$ & 3.5 & 7.7 & 2.2 \\
\hline
\end{tabular}

See text.

is given by the relation (Cox and Lewis, 1966; Smalley et al., 1987)

$P(\Delta)=\frac{T}{M} e^{\left(\frac{-\Delta M}{T}\right)}$,

and $C=1$. On the other hand, for a periodic temporal sequence $C=0$. In case of fractal clustering, one has $C>1$ and in general $C \rightarrow \infty$ as the total length of the fractal increases. For the example described above of the GAC set, we get $C=2.6$ for $L_{\text {tot }}=256$ and $C=4.6$ for $L_{\text {tot }}=1024$ (see Table 1).

In the analysis of our catalog we have calculated also the value of $C$ for a better characterization of our data.

\section{Area investigated}

The general stress field affecting the eastern flank of Mt. Etna (Fig. 3) is very complex and is due to the effect of different processes.

(1) the tectonic forces associated with the interaction of the African and the Eurasian plates (Lentini, 1982; McKenzie, 1970).

(2) the uprising of the magma from depth to shallow structures which is strongly controlled by the master regional fault systems (Shaw, 1980; Wilson and Head, 1981; Cristofolini et al., 1987; Ferrucci et al., 1992) characterized by normal and reverse faulting, as indicated by several seismological studies (Scarpa et al., 1974; Gresta et al., 1987).

(3) the role of the gravitational spreading on this 'unbuttressed' sector of the volcano (Borgia et al., 1992; McGuire et al., 1990; Montalto et al., 1996).

In particular the low eastern flank (Fig. 4) plays a key role in the geodynamic framework of Mt. Etna 
Mt. Etna Volcano ${ }^{-2}$

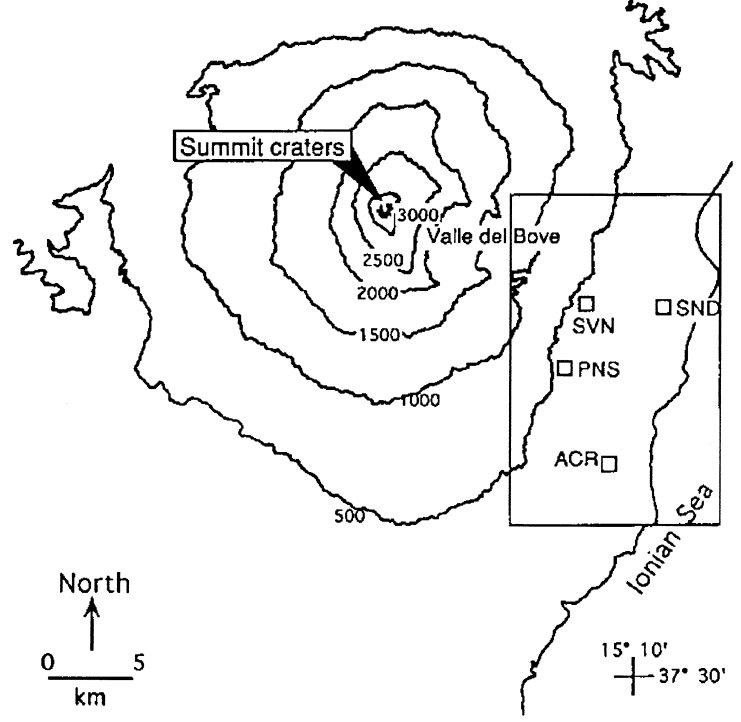

Fig. 3. Map view of the position of the seismic stations and the sector of the eastern flank studied.

volcano. This area is cut by an array of fault escarpments ('Timpe') which define a seismogenic zone of NNW trending faults. The two main structural 'Timpe' domains are the S. Tecla fault and the S. Leonardello fault system.

Morphological, stratigraphic and structural analyses along the Timpe fault zone demonstrate that the faults within it are characterised mainly by both normal and dextral components of slip and that this area has been affected by a general uplift during Holocene (Lo Giudice et al., 1982; Stewart et al., 1993; Valensise and Pantosti, 1992).

Moreover the Timpe zone tectonics seem to be still very active, as revealed by measurements with short geodetic (EDM) networks (Stewart et al., 1993) and by earthquakes occurrence. Analyses of recent seismicity affecting this area (Azzaro et al., 1989; Lo Giudice and Rasá, 1992; Patanè et al., 1994; Montalto et al., 1996) suggest that the S. Tecla fault is deeply involved in tectonic processes and showed that the earthquakes with both deep and shallow foci are mainly related to the S. Tecla fault (trending NW-SE). On the contrary the S. Leonardello fault system (which trends NNW-SSE) is affected only by shallow hypocentres, and it is supposed that the seismicity is associated to the eastward migration of unsupported rigid blocks upon shallow independent decollements.

Finally, in this heterogeneous framework the accumulated stress is very low and it is usually released through several earthquakes of low magnitude $(M \leq 4)$, and 'creep' phenomena, i.e., slow movements producing shallow cracks that do not cause seismicity. Thus the deformation around the Mt. Etna originates from both brittle failure inducing earthquakes and ductile behavior associated with creep, as shown in previous papers (Patanè et al., 1994). The network of the Osservatorio Sismologico di Protezione Civile of Acireale consists of four seismographs equipped with geophons with vertical component S-13 Teledyne and Mark L4C with an oscillation period of $1 \mathrm{~s}$ and a damping equal to 0.7 . Seismic signals are transmitted via cable to the Acireale centre.

Events characterized by $\mathrm{S}-\mathrm{P}$ waves time arrival difference less than $1.5 \mathrm{~s}$ have been considered, this guarantees that earthquakes are located inside the network, i.e., they affect the structural systems investigated.

Two main types of events have been recognized: 1. earthquakes recorded by all the four stations $(M$ $\geq 2$ ),

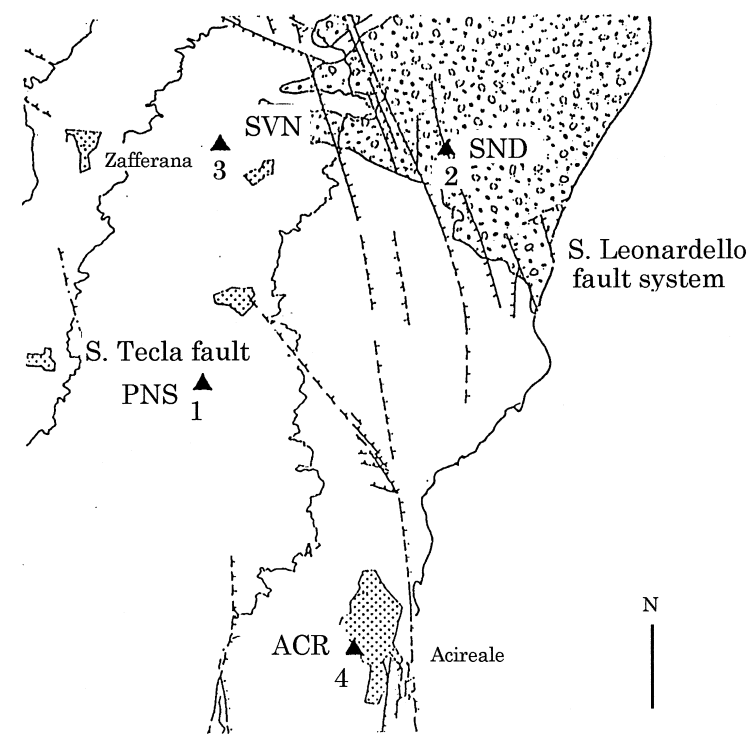

Fig. 4. Structural map of the area investigated (From Lo Giudice et al., 1982). Magnification of the framed area in Fig. 3. 
2. microearthquakes $(1 \leq M \leq 2)$, i.e., single station detections recorded only by the station nearby the relevant fault, occurred during 1989-1994.

Our intention, in the following part of the paper, is to investigate the clustering properties of these second type of events (microearthquakes) which are due neither to a pure brittle nor to a ductile failure; and to quantify the eventual influence of the two main eruptions occurrence (1989 and 1991-1993) on the microearthquakes distribution.

The error bars in the location of the events recorded by the all four stations prevent a reliable investigation and are not considered in this paper.

\section{Discussion}

In this paragraph, we consider the microearthquakes data set recorded by the four stations of the Osservatorio Sismologico di Protezione Civile of Acireale.

The local magnitude of the events was calculated by means of the following relation, using the coda duration estimated from the paper drum recordings at station ACR

$m=2.36 \log (d)-1.57$,

where $d$ is the coda duration in seconds.

The total number $M$ of detected microearthquakes during 1989-1994 is reported in the first column of Table 2 for the four stations. The temporal sequences of the single station recorded microearthquakes ( $M$ $\leq 2)$ are shown in Fig. 5. Seismic swarms are evident, especially for stations 1 and 2 around the two eruption onsets (11th September 1989 and 14 December 1991). The latter are indicated by two vertical dashed lines.

In order to study the behavior of the real temporal series, we simulated periodic and random sequences with the same number of registered events. To create a periodic series the $M$ events were placed at a uniform distance on the total time interval, while for the random series the events were distributed according to uniform random probability.

The first analysis we performed was the calculation of the coefficient of variation $C$ defined in Eq. (7). In Fig. 6 we compare the intercurrence time interval between two consecutive events for the real
Table 2

Values of $M, D,\langle\Delta\rangle, \sigma$ and $C$ for the seismic catalog investigated

\begin{tabular}{lccrrl}
\hline Station & $M$ & \multicolumn{1}{l}{$D$} & $\langle\Delta\rangle$ & \multicolumn{1}{l}{$\sigma$} & $C$ \\
\hline \multicolumn{5}{l}{ Total period (1989-1994) $T=2081$} & days \\
1 & 375 & $0.20 \pm 0.05$ & 5.4 & 9.2 & 1.7 \\
2 & 300 & $0.16 \pm 0.05$ & 6.5 & 15.3 & 2.3 \\
3 & 133 & $0.09 \pm 0.05$ & 15.5 & 29.4 & 1.9 \\
4 & 97 & $0.00 \pm 0.05$ & 20.9 & 29.0 & 1.4 \\
\multicolumn{7}{l}{ Period I (1989-1991) $T=985$ days } & & & \\
1 & 263 & $0.29 \pm 0.05$ & 3.7 & 7.6 & 2.1 \\
2 & 204 & $0.20 \pm 0.05$ & 4.8 & 11.5 & 2.4 \\
3 & 72 & $0.15 \pm 0.05$ & 13.6 & 25.6 & 1.9 \\
4 & 55 & $0.03 \pm 0.05$ & 17.9 & 27.0 & 1.5 \\
\multicolumn{7}{l}{ Period II (1992-1994) $T=1095$ days } & & \\
1 & 112 & $0.07 \pm 0.05$ & 18.2 & 95.1 & 5.2 \\
2 & 96 & $0.07 \pm 0.05$ & 20.4 & 101.4 & 5.0 \\
3 & 61 & $0.03 \pm 0.05$ & 33.8 & 127.5 & 3.8 \\
4 & 42 & $0.00 \pm 0.05$ & 48.2 & 149.8 & 3.1 \\
\hline
\end{tabular}

See text.

temporal distribution of microearthquakes recorded by station 1 (a), the simulated random one (b) and periodic distribution (c) using the same number of events. The real distribution shows a peak at $\Delta=0$, representing multiple shocks on the same day and is characterized by a very long tail. This is numerically demonstrated by the value of the coefficient $C$. In fact we get $C=1.7$. On the other hand for the random distribution which is more centered around the average time interval $\langle\Delta\rangle$ we find $C=1$. This is the value one expects for the probability distribution (8). Finally, for the periodic series (c) we get $C=0$. Therefore, this is a first quantitative indication that the real sequence is not periodic nor random. The same is true for the other stations (see Table 2).

Fig. 6 and the calculation of $C$ give already a measure of the time clustering properties of the earthquakes of our catalog. In order to confirm this observation, we further performed the fractal analysis explained in Section 2. In Fig. 7 we report the natural logarithm of the occupancy probability $P(\tau)$, i.e., the ratio between the occupied and the total number of intervals calculated according to Smalley et al. (1987), as a function of $\ln (\tau), \tau$ being the size of the time interval used to study the distribution for the four stations. The full lines refer to the real 


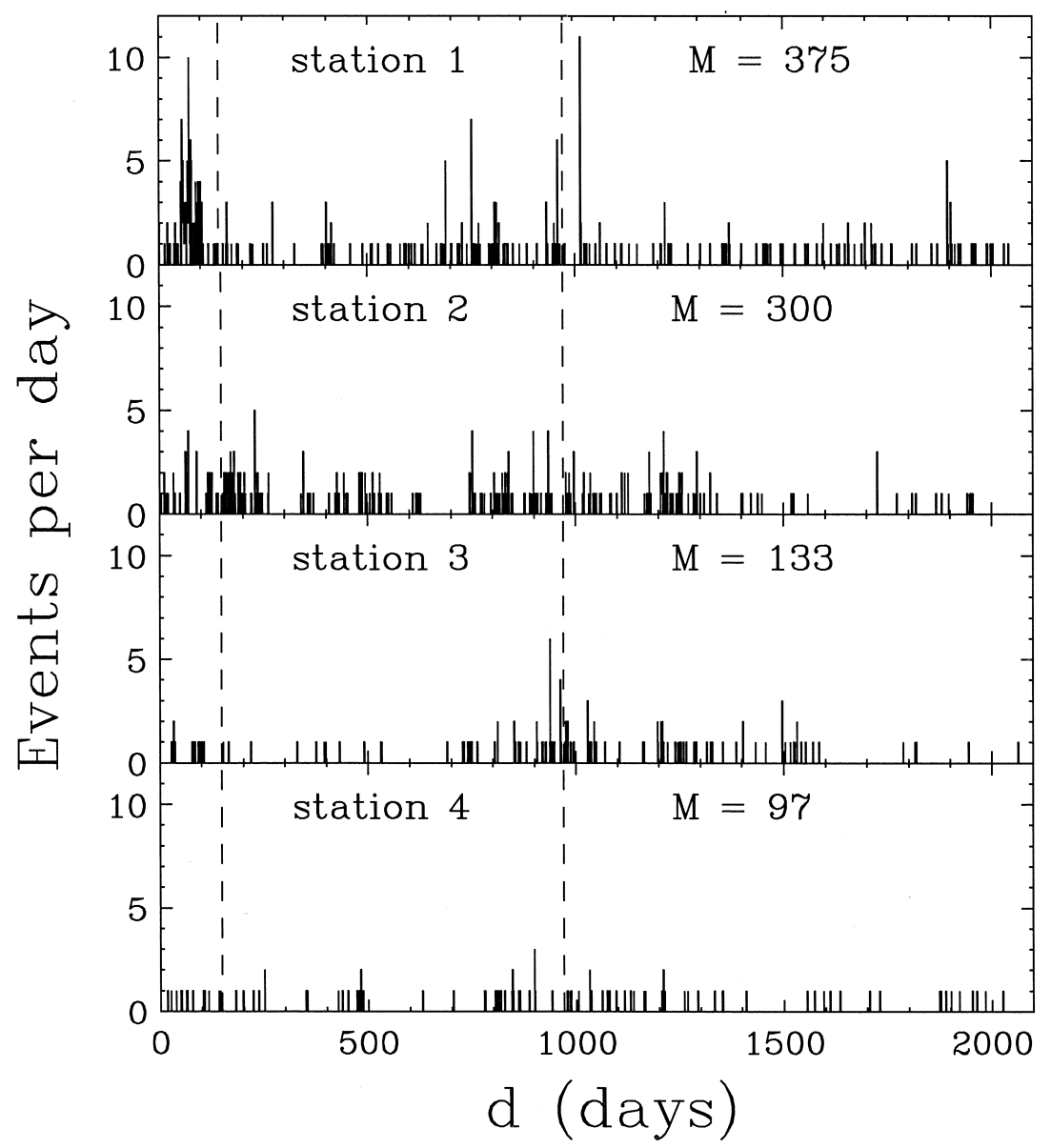

Fig. 5. Daily number of microearthquakes recorded at each station: 1 (PNS) 2 (SND), 3 (SVN) and 4 (ACR). The labels 1 to 4 refer to the four stations of Fig. 4. The vertical dashed lines mark the beginning of eruptions.

sequences, while the dashed ones indicated with $\mathrm{P}$ and $\mathrm{R}$ refer to the simulated periodic and random distributions. It is immediately evident that, excluding station 4, Fig. 7d, the temporal distribution of microearthquakes has not a periodic nor a random character. A clear linear behavior exists in the range $0 \leq \ln (\tau) \leq 2.5$, that is in between 1 and 12 days. It can be very well fitted with a straight (dashed) line whose slope is $1-D$. In other words, one observes a scale-invariant fractal clustering with a fractal dimension ranging from 0.09 to 0.2 . The time interval for the linear fit was chosen by minimizing the error of the fit and changing the maximum time interval in between 6 and 15 days - unfortunately our data prevented us from studying time intervals smaller than 1 day. This procedure allowed us to estimate the error reported in Fig. 7.

The number of events and the total length of the time interval are very close to that of the GAC set of Fig. 2, for which the box counting gives reliable fractal dimension within the errors. So the finite size of the data set seems to be under control. A fit of the random and periodic sequences gives $D=0$ as it should be for the smaller time intervals.

Actually, at variance with the GAC set previously illustrated, the power law is found only in a limited interval range. This is probably due to the influence of an additive random noise which interferes with the fractal scale invariant clustering. In order to study this effect, we added a random noise to the 


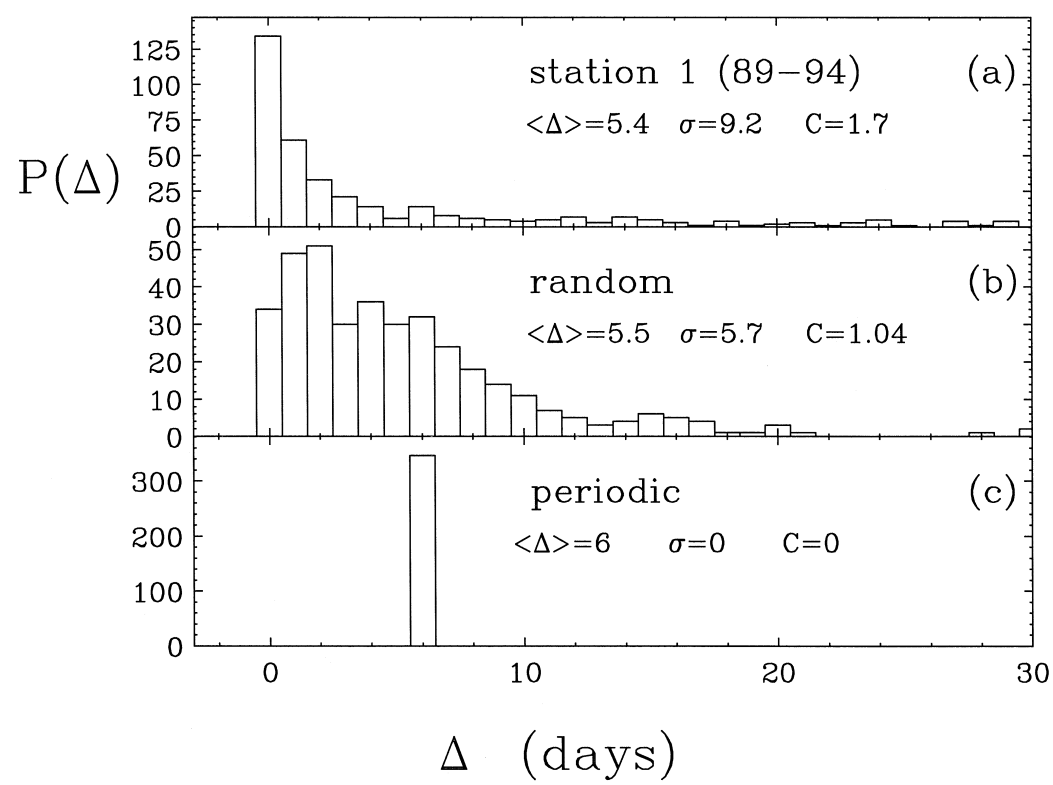

Fig. 6. Intercurrence time probability $P(\Delta)$ for the real distribution of Fig. 5 (a), the synthetic random one (b) and the periodic one (c). The corresponding values of $\langle\Delta\rangle, \sigma$ and $C$ are also reported.

Total Period (89-94)

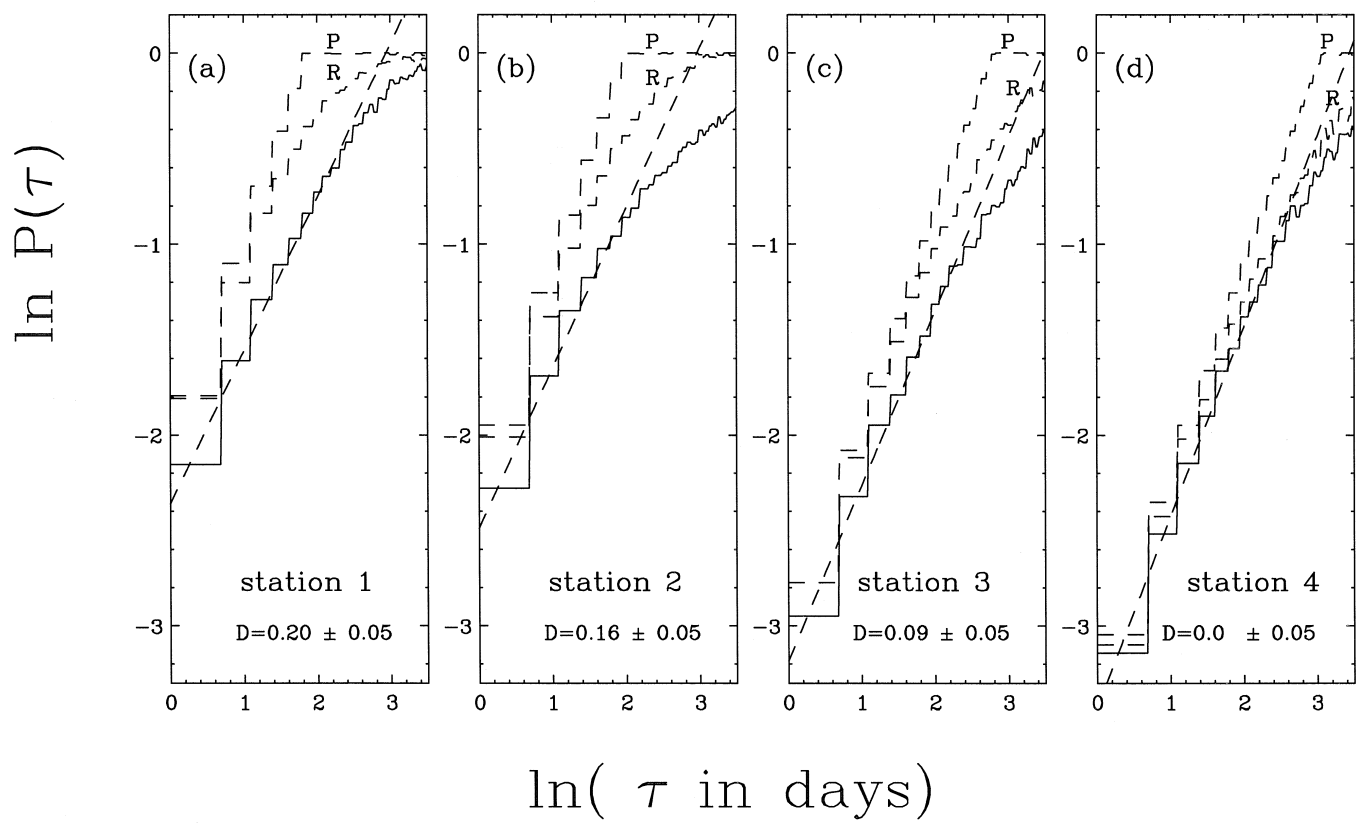

Fig. 7. Plot of $\ln (P(\tau))$ vs. $\ln (\tau)$ in days for the four real distributions of Fig. 5: real earthquakes sequences (full line), random (R dashed line) and periodic (P dashed line). The straight dashed lines are the linear fits to the real sequences, which slope is $1-D$. The correspondent $D$ is also reported with the respective error. 
GAC fractal set of Figs. 1 and 2 and repeated the analysis. In Fig. 8 we show the behavior of the GAC set, generated at the 5th iteration, plus a small amount of random events equal to $\delta_{\text {noise }}=10 \% M$ part (a) and $\delta_{\text {noise }}=20 \% M$ part (b). The curve is indicated by the letters $\mathrm{F}+\mathrm{N}$. For comparison the true fractal curve of Fig. 2 (F) with the fit (dashed line), the periodic and the random curves ( $\mathrm{P}$ and $\mathrm{R}$ respectively) are drawn. The figure clearly shows that the addition of random events has two main effects: (i) the range of the power law reduces by almost three decades (see the fit reported as dashed line), (ii) the value of the fractal dimension is also slightly reduced. When calculating the coefficient $C$ one passes from 4.6 for the real fractal GAC set to 2.5 and 2.2 when the random noise amounts to $10 \%$ and $20 \%$ respectively. These values are very similar to what one gets in our case, see Table 1. Therefore we can draw the first conclusion of our investigation. The microseismicity recorded by our network shows sig- nal of fractal clustering with a probable addition of a small amount of spurious random noise.

A previous analysis performed on the same data by means of the correlation functions method (Vinciguerra et al., 1998) showed an influence of the two eruptions on the seismic activity recorded by station 1 and station 2. In order to verify if the microearthquake fractal clustering is also influenced by the two eruption occurrences, we repeated our analysis considering two subperiods. Our data were available from April 1989 to December 1994 and the two eruptions occurred on 11th September 1989 and 14th December 1991. Therefore we distinguished the period between 22 April 1989 and 31 December 1991 which includes the two eruptions onsets shown in Fig. 5 (period I) and the following period (period II), which goes from the beginning of 1992 to the end of 1994. The two periods have almost the same total length, that is $T=985$ and $T=1095$ days respectively. The fractal analysis is similar to that

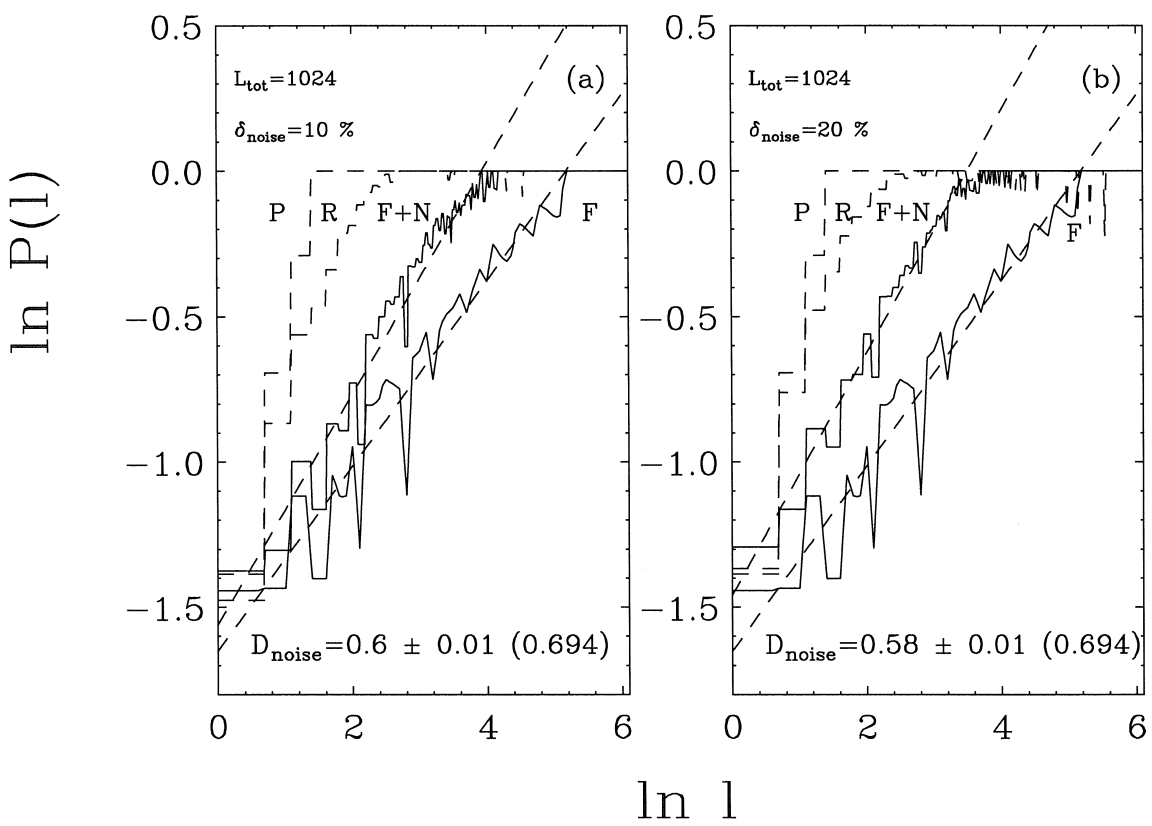

Fig. 8. In this figure the effect of a random noise on the 5th order GAC set is illustrated. We plot (full curve indicated by $\mathrm{F}+\mathrm{N}$ ) the $\ln (P(l))$ vs. $\ln (l)$ for the GAC set plus a small amount of random events equal to $\delta_{\text {noise }}=10 \% M$ (a) and $\delta_{\text {noise }}=20 \% M(\mathrm{~b})$. The curve of the GAC set without noise (F) and its relative fit is also reported for comparison. As done in the previous figures we plot also the periodic (P) and random sequence (R) of events for the same total number of records. The effect of noise reduces the range of validity of the power law and at the same time slightly diminishes the value of the fractal dimension. The latter is also reported in comparison with the true value in parenthesis. See text for further details. 
already discussed for the total period of observation and is illustrated in Figs. 9 and 10.

A clear fractal clustering is observed for the station 1-3 in the period I and the fractal dimension tends to increase slightly Fig. 11. The values of the coefficient $C$ tend to increase too, though the total time interval was halved. This is a further indication of the fractal character of the distribution in the presence of eruption onsets.

On the other hand a drastic reduction of the fractal dimension is found for the period II, when no eruption onsets are present. In this case the distinction between fractal and random behavior is very weak.

In Fig. 11 the value of $D$ for the four stations is plotted for all the periods investigated. It allows to draw the following considerations.

(i) The fractal analysis and the calculation of $C$ for the two subperiods seem to indicate a strong correlation between the eruptions and the fractal clustering of the microseismic activity. Generally the value of $D$ before the eruptions onset (period I) is greater than the value of $D$ when there are no eruptions (period II). (ii) The increase of the fractal dimension is very evident at station 1 . It means that the microearthquakes clustering features are very different during the two periods analysed and particularly that big microearthquake swarms occur before the eruption onsets as confirmed by other different statistical analysis (Vinciguerra et al., 1998). We can suppose that the magma rise preceding the eruption onsets favours microearthquake occurrence. The microseismicity is the result of the inflation process affecting the volcano edifice (Hill, 1977; Bonaccorso et al., 1990; Budetta et al., 1989; Ferrucci et al., 1993). In this framework the Timpa di S. Tecla is very active, as analyses of its recent seismic activity underline, and affected by both deep and shallow tectonics (Patanè et al., 1994; Montalto et al., 1996). This structure is closely related to the magma transfer processes occurring before the eruption onsets and seems to control the magma rise inside the volcanic edifice. The inflation process induces, at shallow levels, local instability phenomena provoking microearthquake swarms with a fractal clustering.

(iii) The $D$ increase is less marked at the station 2 and 3. This is because the S. Leonardello fault

Period I (89-91)

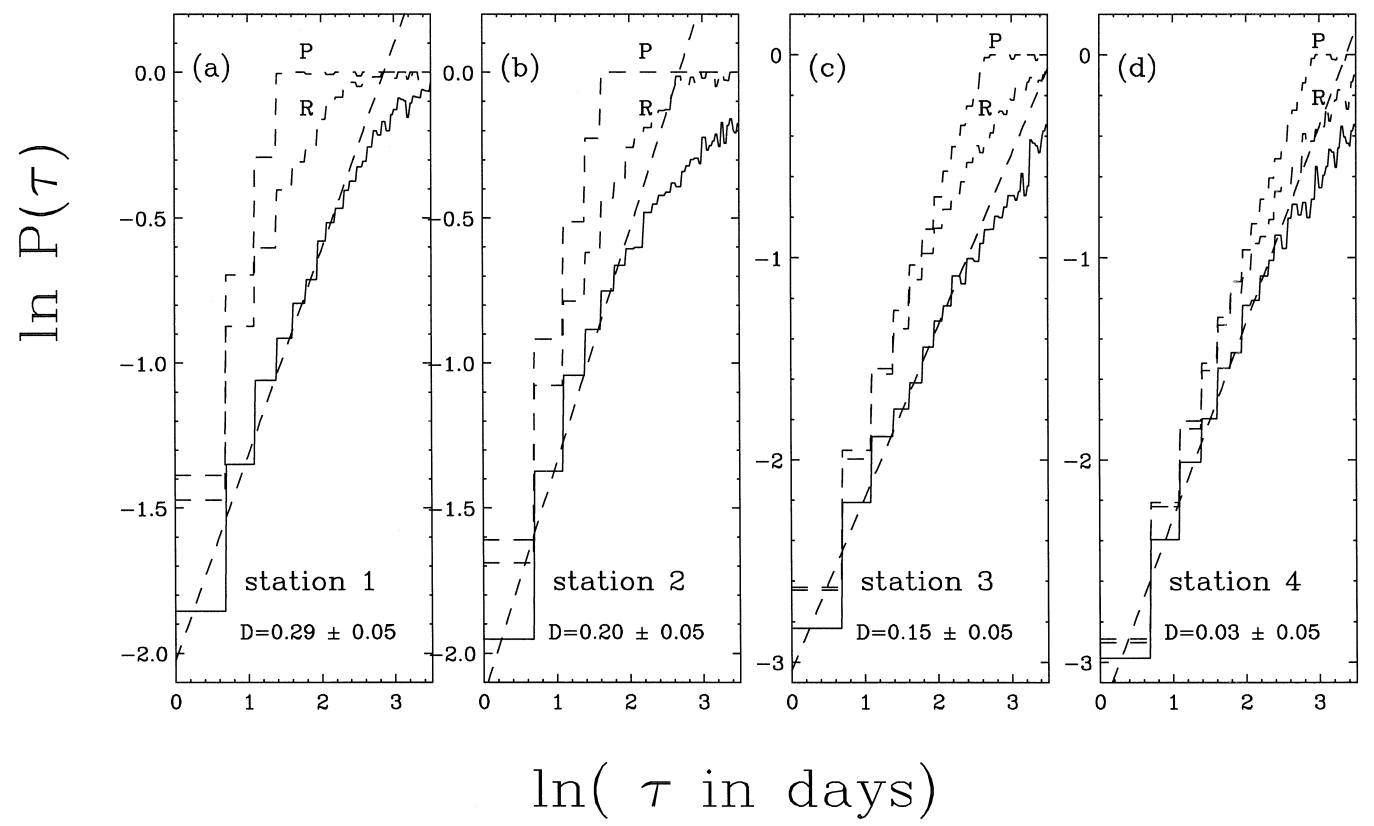

Fig. 9. The same as Fig. 7 but for the period I (1989-1991) which includes the two major eruption onsets shown in Fig. 5. See text. 


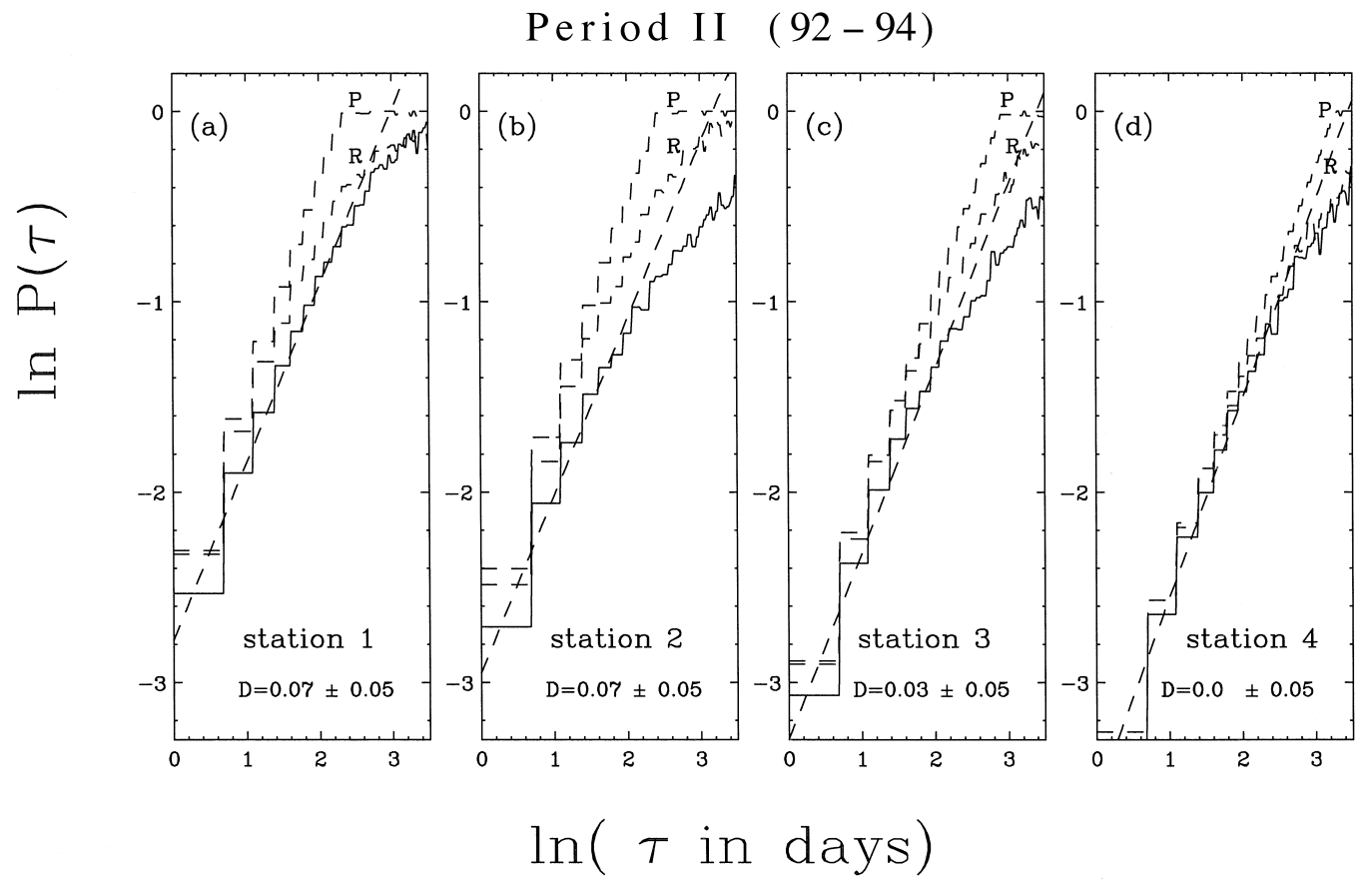

Fig. 10. The same as Fig. 7 and Fig. 9 but for the period II (1992-1994), which does not include the two major eruption onsets. See text.

systems and the other secondary structural systems are affected only by shallow distensive tectonic processes and they do not play an active role on the magma transfer as the S. Tecla fault. Nevertheless the stress-field variation originated by the magma ascent involves these structures; This stress-field

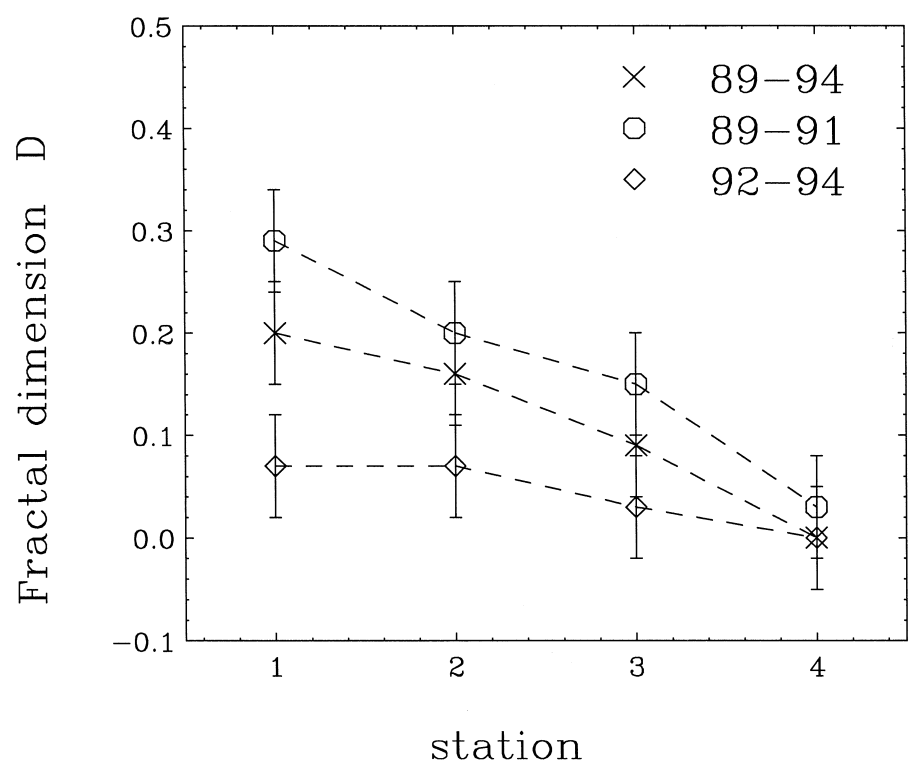

Fig. 11. The values of the fractal dimensions are plotted for the periods studied. Dashed lines are drawn to guide the eye. 
variation can favour a local shallow sliding process and can induce in the region of station 2 and 3 local detachments and consequently a microseismicity phenomenon less important than the previous case of station 1.

(iv) No appreciable increase in the $D$ value of the station 4 seems to occur in correspondence with the eruptions. The $D$ value is always very close to the random noise. Actually the timpa di Acireale plays a secondary role in the local stress-field evolution as showed by the low number of microearthquakes. The timpa di Acireale is not influenced by the eruptions onsets and the microseismicity time distribution does not change its clustering properties during the period I and II.

\section{Conclusions}

In this paper we presented a detailed analysis of the fractal properties of microseismicity affecting the low eastern flank of Mt. Etna volcano during the period 1989-1994. The fractal clustering is very probably linked to the onset of the two eruptive phenomena occurred in the same period, confirming a recent result obtained with the correlation functions approach (Vinciguerra et al., 1998).

More precisely the following conclusions can be drawn.

(1) The temporal series of the microearthquakes recorded at the stations 1-3 are neither periodic nor random, but they show a scale invariant behavior as confirmed also by the value of the coefficient of variation C. Considering the whole period (19891994 a fractal clustering was found with a fractal dimension $D$ ranging from 0.09 to 0.20 in a time interval between 1 day and 12 days.

In the period II (1992-1994), when no eruption onsets occur, the temporal series are consistent with a random noise.

In the period I (1989-1991), when the two eruptions occurred (11th September 1989 and 14th December 1991) the fractal clustering is evident and an increase in the value of $D$ with respect to the whole period (1989-1994) was in general found for stations $1-3$. The increase of $D$ is more marked for the station 1 , but it is appreciable for the stations 2 and 3 ; there is no increase for the station 4 , whose seismicity is always consistent with a random noise. This suggests a strong relation of the $\mathrm{S}$. Tecla fault with the geodynamic evolution of the volcano edifice, in proximity of an eruption occurrence. The S. Leonardello fault systems and the other secondary structural systems are not so deeply involved by the magma arise, but they feel, at a shallow level, the local stress variations. Microearthquakes clustering affect prevalently the S. Tecla Timpa fault and, in a secondary way, the S. Leonardello fault system.

(2) The analysis should not be drastically affected by the limited number of the events. A strong support in this sense comes from the analysis performed on the GAC set. In fact, this fractal distribution is very generic and has no peculiar aspect which could bias the comparison with the real time series. However the power law behavior seem to be affected by an additive random noise, which probably reduces the range of the scaling behavior. This spurious noise influences the exact estimate of $D$. Again the example of the GAC set plus noise suggests that the error should be very small (of the order of a few percent). On the other hand, one is more interested in the relative strong uprising of $D$, which could be an important feature that characterizes the local seismicity before eruptions.

\section{Acknowledgements}

We would like to thank Dr. D.J. Varnes and the other anonymous referees for their critical reading of the manuscript and their useful comments.

\section{References}

Azzaro, R., Carveni, P., Lo Giudice, E., Rasá, R. II, 1989. Terremoto di Codavolpe (basso versante orientale Etneo) del 29 Gennaio: Campo macrosismico e fratturazione cosismica. Boll. GNV-CNR 1, 1-12.

Bonaccorso, A., Campisi, O., Falzone, G., Puglisi, B., Velardita, R., Villari, L., 1990. Etna: Ground deformation: geodimeter trilateration and borehole tiltmetry. In: Barberi, F., Bertagnini, A., Landi, P. (Eds.), Mt. Etna 1989 eruption. CNR-GNV Special Issue, 44-47.

Budetta, G., Grimaldi, M., Luongo, G., 1989. Variazioni temporali di gravità all'Etna. Boll. G.N.V. 5 (1), 107-117.

Borgia, A., Ferrari, L., Pasquarè, G., 1992. Importance of gravitational spreading in the tectonic and volcanic evolution of Mount Etna. Nature 357, 231-235. 
Cox, D.R., Lewis, P.A.W., 1966. The statistical analysis of series of events. Methuen, London, England.

Cristofolini, R., Gresta, S., Imposa, S., Patanè, G., 1987. Feeding mechanism of eruptive activity at Mt. Etna based on seismological and petrological data. In: King, C.-Y., Scarpa, R. (Eds.), Modelling of Volcanic Processes. Earth Evol. Sci., pp. 73-93.

Ferrucci F., Gresta S., Patanè D., Rasá R., 1992. Inferences on the magma feeding system at Mt. Etna Volcano from seismological, structural and volcanological data. Proc. XI National Meeting GNGTS, 1, 455-460.

Ferrucci, F., Rasá, R., Gaudiosi, G., Azzaro, R., Imposa, S., 1993. Mt. Etna: A model for the 1989 eruption. J. Volc. Geoth. Res. $56,35-55$.

Gresta, S., Imposa, S., Patanè, D., Patanè, G., 1987. Volcanic tremor at Mt. Etna: state of the art and perspectives. Pu. Appl. Geophys. 125, 1079-1095.

Hill, D.P., 1977. A model for earthquake swarms. Journal of Geophysical Research 82, 1347-1352.

Kagan, Y.Y., Jackson, D.D., 1991. Long-term earthquake clustering. Geophys. J. Int. 104, 117-133.

Lentini, F., 1982. The Geology of the Mt. Etna Basement. In: Romano, R. (Ed.), Mount Etna Volcano. Mem. Soc. Geol. It., pp. 7-25.

Lo Giudice, E., Patanè, G., Rasá, R., Romano, R., 1982. The structural framework of Mount Etna. In: Romano, R. (Ed.), Mount Etna Volcano. Mem. Soc. Geol. Ital., 23, 125-158.

Lo Giudice, E., Rasá, R., 1992. Very shallow earthquakes and brittle deformation in active volcanic areas: the etnean region as example. Tectonophys. 202, 257-268.

Mandelbrot, B.B., 1967. How long is the coast of Britain? Statistical self-similarity and fractional dimension. Science 156, 636638.

McGuire, W.J., Pullen, A.D., Saunders, S.J., 1990. Recent dykeinduced large-scale block movement at Mount Etna and potential slope failure. Nature 343, 357-363.

McKenzie, D.P., 1970. The plate tectonics of the Mediterranean region. Nature 226, 239-243.
Montalto, A., Vinciguerra, S., Menza, S., Patanè, G., 1996. Recent seismicity of Mount Etna: implications for flank instability. In: McGuire, W.J., Jones, A.P., Neuberg, J. (Eds.), Volcano Instability on the Earth and Other Planets, Geological Society Special Publication No. 110, pp. 169-177.

Patanè, G., Montalto, A., Imposa, S., Menza, S., 1994. The role of regional tectonics, magma pressure and gravitational spreading in earthquakes of the eastern sector of Mt. Etna Volcano. J. Vol. Geotherm. Res. 61, 253-266.

Scarpa, R., Patanè, G., Lombardo, G., 1974. Space-time evolution of seismic activity at Mount Etna during 1974-1982. Ann. Geophys. 16, 451-462.

Shaw, H.R., 1980. The fracture mechanism of magma transport from the mantle to the surface. In: Physics of Magmatic Processes. Princetown Univ. Press, Princetown, NJ, pp. $203-$ 263.

Smalley, R.F. Jr., Chatelain, J.L., Turcotte, D.L., Prevot, R., 1987. A Fractal approach to the clustering of earthquakes: applications to the seismicity of the New Hebrides. Bull. Seism. Soc. Am. 77 (4), 1368-1381.

Stewart, I., McGuire, W., Vita-Finzi, C., Firth, C., Holmes, R., Saunders, S., 1993. Active faulting and neotectonic deformation on the eastern flank of Mount Etna Sicily. Z. Geomorph. N.E. 94, 73-94, Suppl. -Bd.

Tél, T., Fülöp, A., Vicsek, T., 1989. Determination of fractal dimensions for geometrical multifractals. Physica A 159, 155166.

Valensise, G., Pantosti, D., 1992. A 125 Kyr-long geological record of seismic source repeatability: the Messina Straits (southern Italy) and the 1908 earthquake (Ms 7.5). Terra Nova 4, 472-483.

Vinciguerra, S., Latora, V., Vinciguerra, D., 1998. Time correlation of the microseismic activity of the low eastern flank of Mt. Etna. Pu. Appl. Geophys. 152, 165-174.

Wilson, L., Head, S.W. III, 1981. Ascent and eruption of basaltic magma on the Earth and the Moon. J. Geophys. Res. 86, 2971-3001. 